\title{
The effect of rosuvastatin on thromboinflammation in the setting of acute coronary syndrome
}

\author{
Travis R. Sexton • Eric L. Wallace - Tracy E. Macaulay • Richard J. Charnigo • \\ Virgilio Evangelista $\cdot$ Charles L. Campbell $\cdot$ Alison L. Bailey $\cdot$ Susan S. Smyth
}

Published online: 12 October 2014

(C) The Author(s) 2014. This article is published with open access at Springerlink.com

\begin{abstract}
In patients with acute coronary syndromes (ACS), early therapy with high-dose statins may reduce short-term adverse clinical outcomes. The mechanisms responsible are not known but could involve anti-inflammatory or anti-thrombotic effects. Compelling evidence from experimental models and clinical studies suggests that the interplay between inflammatory and thrombotic systems, typified by platelet-monocyte and platelet-neutrophil interactions, might be a key regulator of ischemic vascular events. The study sought to determine if early, high-dose administration of the HMG-CoA reductase inhibitor rosuvastatin in the setting of ACS exerts beneficial vascular effects by reducing, and inhibiting biomarkers of thromboinflammation, such as platelet-monocyte and plateletneutrophil interactions, and biomarkers of myocardial necrosis. A total of 54 patients presenting with ACS within $8 \mathrm{~h}$ of symptom onset were randomized to rosuvastatin $40 \mathrm{mg}$ or placebo. Rosuvastatin significantly reduced interactions between platelets and circulating neutrophils $(P=0.015)$ and monocytes $(P=0.009)$ within $24 \mathrm{~h}$. No
\end{abstract}

Clinical Trial Registration: http://clinicaltrials.gov/ct2/show/ NCT01241903?term=Smyth\&rank=19

Electronic supplementary material The online version of this article (doi:10.1007/s11239-014-1142-x) contains supplementary material, which is available to authorized users.

T. R. Sexton - E. L. Wallace - T. E. Macaulay ·

R. J. Charnigo · C. L. Campbell · A. L. Bailey ·

S. S. Smyth $(\bowtie)$

The Gill Heart Institute, University of Kentucky, BBSRB B201,

Lexington, KY 40506, USA

e-mail: SusanSmyth@uky.edu

V. Evangelista

Mario Negri Sud, Santa Maria Imbaro, Chieti, Italy significant effects were observed on platelet aggregation or plasma levels of PF4, sP-selectin, or sCD40L, whereas significant reductions of RANTES occurred over time in both treatment groups. Plasma levels of myeloperoxidase (MPO) declined more rapidly with rosuvastatin therapy than placebo. In a subset of patients with normal cardiac necrosis biomarkers at randomization, rosuvastatin therapy was associated with less myocardial damage as measured by troponin-I or CK-MB. Early administration of high-dose statin therapy in patients with ACS appears to improve biomarkers of inflammation within $8 \mathrm{~h}$, which may translate into fewer ischemic events.

Keywords Acute coronary syndrome $\cdot$ Acute myocardial infarction · Platelets · Statin · Thromboinflammation

\section{Introduction}

Platelets play a central role in the pathogenesis of acute coronary syndromes (ACS) and in thrombotic complications of percutaneous coronary intervention (PCI). Antiplatelet therapy is a mainstay of the initial management of patients with non-ST-segment elevation (NSTE)-ACS, STsegment elevation myocardial infarction (STEMI), and those undergoing PCI [1]. However, despite the general use of anti-platelet agents in ACS, recurrent ischemic events occur in a substantial number of patients. ACS is accompanied by an intense inflammatory response. Compelling evidence from experimental models and clinical studies suggests that interplay between the inflammatory and thrombotic systems might be a key regulator of ischemic vascular events.

Platelet-leukocyte interactions offer an important mechanistic link between the inflammatory and thrombotic 
systems. Activated platelets bind to circulating leukocytes and recruit them to sites of vascular injury and to thrombus. Experiments in preclinical models indicate that the interactions contribute to local, downstream, and systemic injury responses [2]. In both ACS and PCI, high levels of circulating platelet-leukocyte heterotypic aggregates correlate with markers of myocardial necrosis. Moreover, interventions to block the interactions may reduce ischemic injury. For example, in the recently reported SELECT-ACS trial, the P-selectin antagonist inclacumab, which blocks the ability of platelets to bind to leukocytes [3], reduced cardiac biomarkers of necrosis (troponin-I and creatine kinase-MB [CK-MB]) in patients with ACS [4]. Such findings suggest that interactions between platelets and leukocytes may contribute to ischemic tissue injury in the setting of ACS.

In this study, we sought to determine if administration of a high-dose of the 3-hydroxy-3-methylglutaryl coenzyme A (HMG-CoA) reductase inhibitor (statin) rosuvastatin early in the setting of ACS would exert beneficial effects by reducing thromboinflammation, specifically platelet interactions with monocytes and neutrophils. We selected rosuvastatin because of its ability to lower inflammatory markers in addition to low-density lipoprotein (LDL)-cholesterol. In the JUPITER trial [5], which examined rosuvastatin as primary prevention in patients with increased levels of high-sensitivity C-reactive protein (hs-CRP), the largest benefit occurred in subjects who had lowering of both hs-CRP and LDL-cholesterol levels [6]. Several studies have suggested that the benefits of high dose statin therapy may begin prior to any significant blood cholesterol lowering effect $[7,8]$. In these trials, rosuvastatin or atorvastatin reduced peri-procedural cardiac necrosis in stable coronary artery disease and ACS even when administered less than $24 \mathrm{~h}$ prior to the procedure [9-12].

To provide mechanistic insight into the actions of early, high dose statin therapy, we enrolled ACS patients who presented within $8 \mathrm{~h}$ of symptom onset and randomized them to receive a $40 \mathrm{mg}$ dose of rosuvastatin or placebo and monitored monocyte-platelet and neutrophil-platelet aggregates, markers of platelet activation and inflammation, within the first $24 \mathrm{~h}$. Our findings suggest that administration of high-dose rosuvastatin early in the course of ACS reduces interactions of platelets with monocytes and neutrophils within $8 \mathrm{~h}$ and thereby exert a beneficial effect on thromboinflammatory pathways.

\section{Materials and methods}

Patients and study design

The early use of rosuvastatin in acute coronary syndromes: targeting platelet-leukocyte interactions (AVATAR) trial enrolled patients presenting to the University of Kentucky hospitals with (1) cardiac ischemia within the last $8 \mathrm{~h}$, (2) biomarker evidence of cardiac ischemia and/or (3) electrocardiographic evidence of cardiac ischemia. At screening, patients 18-80 years of age had to either not be taking a statin medication or be on a low dose of a statin (defined as pravastatin $\leq 40 \mathrm{mg}$, simvastatin $\leq 20 \mathrm{mg}$, or atorvastatin $\leq 10 \mathrm{mg}$ ). Supplemental Table 1 lists additional inclusion and exclusion criteria.

This was an investigator-initiated trial designed by the investigators (www.clinicaltrials.gov, trial NCT01241903). The institutional review board at the University of Kentucky approved the protocol, and all patients provided written informed consent before entering the trial. Patients were enrolled in a double-blind manner and were randomized to high-dose rosuvastatin $(40 \mathrm{mg})$ or a placebo given immediately upon consent. All patients received rosuvastatin $20 \mathrm{mg}$ after the first hospital day and daily thereafter for 30 days. Blood was sampled at baseline, prior to randomization, and at approximately 8 and $24 \mathrm{~h}$ following the initial dose. Study follow-up occurred daily while in hospital and at 30 days after enrollment. Major adverse cardiovascular events were collected at 30 days.

\section{Flow cytometry}

See supplemental materials for details.

Platelet aggregation

See supplemental materials for details.

Biomarker assays

See supplemental materials for details.

Clinical outcomes

See supplemental materials for details.

Statistical analyses

See supplemental materials for details.

\section{Results}

Patient characteristics

From November 29, 2011, through July 24, 2013, 54 patients were enrolled and 53 received either placebo $(n=26)$ or a $40 \mathrm{mg}$ dose of rosuvastatin $(n=27)$ at study entry. One enrolled patient refused to take the initial dose of study 
Table 1 Baseline characteristics of AVATAR subjects

\begin{tabular}{|c|c|c|c|}
\hline & Placebo & Rosuvastatin & $P$ value \\
\hline \multicolumn{4}{|l|}{ Demographics } \\
\hline Men & $17(65 \%)$ & $16(59 \%)$ & 0.779 \\
\hline Women & $9(35 \%)$ & $11(41 \%)$ & 0.779 \\
\hline Age & $52.8(10.7)$ & $57.2(10.4)$ & 0.142 \\
\hline Caucasian & $22(85 \%)$ & $24(89 \%)$ & 0.704 \\
\hline African American & $3(12 \%)$ & $3(11 \%)$ & 1.000 \\
\hline Hispanic & $1(3 \%)$ & $0(0 \%)$ & 0.491 \\
\hline \multicolumn{4}{|l|}{ Cardiovascular History } \\
\hline Hypertension & $18(69 \%)$ & $17(63 \%)$ & 0.773 \\
\hline History of smoking & $21(81 \%)$ & $23(85 \%)$ & 0.728 \\
\hline Hyperlipidemia & $19(73 \%)$ & $19(70 \%)$ & 1.000 \\
\hline Diabetes & $5(19 \%)$ & $7(26 \%)$ & 0.745 \\
\hline Family history of CHD & $15(58 \%)$ & $14(52 \%)$ & 0.785 \\
\hline Prior MI & $9(35 \%)$ & $5(19 \%)$ & 0.224 \\
\hline \multicolumn{4}{|l|}{ Cardiovascular } \\
\hline Ejection fraction, $(\%)$ & $49.5(10.4)$ & $55.4(7.9)$ & 0.056 \\
\hline
\end{tabular}

Data are $n(\%)$ or mean (SD)

$P$ values for the qualitative variables (sex, race, cardiovascular history) were calculated using Fisher's Exact test

$P$ values for quantitative variables were calculated with a two-sample $t$ test

CHD coronary heart disease, $M I$ myocardial infarction

medication and was removed from further analysis. Table 1 lists demographic and baseline characteristics of the patients and Table 2 lists cardiovascular medications that patients received prior to enrollment and in the $24 \mathrm{~h}$ following randomization. No significant differences in demographics or cardiovascular medication use were noted between the two groups. Table 3 lists clinical features of the patients. Overall, $37(70 \%)$ of the subjects had a non-ST-segment elevation myocardial infarction (NSTEMI) or unstable angina (UA), and $17(30 \%)$ of the patients suffered a STEMI. Of the 26 patients randomized to the placebo group, 10 had STEMI and 16 NSTEMI/UA. Of the 27 patients randomized to the rosuvastatin group, 7 had STEMI and 20 NSTEMI/UA. Forty six of the patients $(86.8 \%)$ underwent PCI while in hospital. The average time from hospital admission to PCI was $498 \pm 156 \mathrm{~min}$ for the placebo group $(n=25)$ and $429 \pm 136$ for rosuvastatin group $(n=21 ; P=0.744)$. $42.3 \%$ of the patients in the placebo group and $48 \%$ in the rosuvastatin group underwent PCI prior to the $8 \mathrm{~h}$ blood draw. No significant differences were observed in clinically obtained troponin-I levels between the placebo and rosuvastatin groups at near baseline or approximately $24 \mathrm{~h}$ after randomization. Additionally, platelet and white blood cell counts at the time of study enrollment and at $24 \mathrm{~h}$ were similar between the two groups.
Table 2 Baseline and $24 \mathrm{~h}$ medications for AVATAR subjects

\begin{tabular}{lccc}
\hline & Placebo & Rosuvastatin & $P$ value \\
\hline Medication prior to dosage & & & \\
Aspirin & $24(92 \%)$ & $24(89 \%)$ & 1.000 \\
P2Y12 inhibitor & $22(85 \%)$ & $23(85 \%)$ & 1.000 \\
Beta blocker & $13(50 \%)$ & $10(37 \%)$ & 0.412 \\
ACE inhibitor & $13(50 \%)$ & $7(26 \%)$ & 0.093 \\
GPI & $4(15 \%)$ & $3(11 \%)$ & 0.704 \\
Heparin & $19(73 \%)$ & $14(52 \%)$ & 0.158 \\
Bivalirudin & $0(0 \%)$ & $1(4 \%)$ & 1.000 \\
Medication within $24 \mathrm{~h}$ & & & \\
following dosage & & & \\
Aspirin & $24(92 \%)$ & $26(96 \%)$ & 0.610 \\
P2Y12 inhibitor & $25(96 \%)$ & $25(93 \%)$ & 1.000 \\
Beta blocker & $17(65 \%)$ & $22(81 \%)$ & 0.224 \\
ACE inhibitor & $16(62 \%)$ & $14(52 \%)$ & 0.583 \\
GPI & $4(15 \%)$ & $3(11 \%)$ & 0.704 \\
Heparin & $19(73 \%)$ & $20(74 \%)$ & 1.000 \\
Bivalirudin & $2(8 \%)$ & $1(4 \%)$ & 0.610 \\
\hline Data $n$ & & &
\end{tabular}

Data are $n(\%)$ or mean (SD)

$P$ values were calculated using Fisher's Exact test GPI glycoprotein IIb/IIIa inhibitor 
Table 3 Clinical characteristics of patients

\begin{tabular}{llll}
\hline & Placebo & Rosuvastatin & $P$ value \\
\hline ACS & & & \\
STEMI & $10(38 \%)$ & $7(22 \%)$ & 0.387 \\
NSTEMI/UA & $16(62 \%)$ & $20(78 \%)$ & 0.387 \\
Hemogram & & $220 \pm 54$ & 0.462 \\
Baseline platelet count & $231 \pm 48$ & $195 \pm 47$ & $0.9 \pm 0.7$ \\
24 h platelet count & $215 \pm 55$ & $9.4 \pm 0.7$ & 0.871 \\
Baseline WBC & $10.1 \pm 0.7$ & & 0.931 \\
24 h WBC & $9.4 \pm 0.7$ & $0.47(0.08-3.11)$ & 0.907 \\
Cardiac Necrosis & & $6.00(0.12-36.26)$ \\
Baseline troponin & $0.45(0.09-2.60)$ & 0.478 \\
Peak troponin & $9.14(0.95-35.37)$ & \\
\hline
\end{tabular}

Data are presented as $n(\%)$, mean $\pm \mathrm{SD}$, or median (25th-75th percentile). STEMI, NSTEMI, and unstable angina (UA) were determined by the attending physician based on ECG and cardiac necrosis biomarker lab results. $P$ values were calculated using Fisher's Exact Test for qualitative variables, a two-sample $t$ test for approximately normally distributed quantitative variables, and a Mann-Whitney rank sum test for other quantitative variables

Table 4 Monocyte-platelet and neutrophil- platelet interactions are decreased significantly following treatment of a high-dose rosuvastatin

\begin{tabular}{llllllll}
\hline & Baseline \% (SE) & $P$ value* & $8 \mathrm{~h} \%(\mathrm{SE})$ & $P$ value $^{\dagger}$ & $24 \mathrm{~h} \%$ (SE) & $P$ value $^{\dagger}$ & Main finding $^{*}$ \\
\hline $\begin{array}{l}\text { Monocyte-platelet } \\
\quad \text { Placebo }\end{array}$ & $49.2(3.6)$ & 0.132 & $46.1(6.4)$ & 0.004 & $51.6(6.2)$ & 0.003 & 0.009 \\
$\quad \begin{array}{l}\text { Rosuvastatin } \\
\text { Neutrophil-platelet }\end{array}$ & $65.5(6.4)$ & & $40.2(4.6)$ & & $40.7(5.6)$ & \\
$\quad$ & & & & & \\
Placebo & $23.1(3.5)$ & 0.197 & $18.6(3.9)$ & 0.009 & $18.1(3.9)$ & 0.033 & 0.015 \\
Rosuvastatin & $31.7(4.4)$ & & $12.8(2.1)$ & & $13.3(2.2)$ & \\
\hline
\end{tabular}

* Linear mixed model comparing treatment groups on baseline values, with Bonferroni adjustment

$\dagger$ Linear mixed model comparing treatment groups on the change from baseline, with Bonferroni adjustment

* Linear mixed model comparing treatment groups overall, across all time points

Effect of early administration of high-dose rosuvastatin on the percentage monocytes and neutrophils

with associated platelets

Early administration of high dose rosuvastatin resulted in a statistically significant reduction in circulating monocyteplatelet aggregates over the first $24 \mathrm{~h}(P=0.0029)$. At the time of study enrollment (baseline), the percentage of monocytes with associated platelets was $65.5 \pm 6.4 \%$ (mean \pm SEM) in the rosuvastatin group and $49.2 \pm 3.6 \%$ in the placebo group ( $P=0.132$ after Bonferroni adjustment; Table 4). At $8 \mathrm{~h}$, a striking $25.3 \%$ absolute reduction in average monocyte-platelet aggregates occurred with rosuvastatin, whereas the placebo group had a modest $3.1 \%$ decrease ( $P=0.0039$ for difference between doses from baseline to $8 \mathrm{~h}$, Table 4). No further decline occurred at $24 \mathrm{~h}$, when monocyte-platelet aggregates averaged $40.7 \pm 5.6 \%$ with rosuvastatin and $51.6 \pm 6.2 \%$ with placebo $(P=0.0029$ for difference between doses from baseline to $24 \mathrm{~h}$, Table 4 ). Figure 1a presents the individual data normalized to baseline (fold change where 1.0 equals baseline value) and demonstrates an overall reduction in the proportion of monocytes with platelets in patients who received early, high dose rosuvastatin.

Analysis of neutrophil-platelet aggregates demonstrated a significant lowering in the rosuvastatin group over the first $24 \mathrm{~h}(P=0.0145$, Table 4$)$. At baseline, $31.7 \pm 4.4 \%$ of the neutrophils had attached platelets in the rosuvastatin group versus $23.1 \pm 3.5 \%$ in the placebo group. At $8 \mathrm{~h}$, the average percentage of circulating neutrophils with adherent platelets was markedly lower in the rosuvastatin group at $12.8 \pm 2.1$ (18.9\% absolute reduction) but only slightly lower in the placebo group at $18.6 \pm 3.9$ (4.5\% absolute reduction) $(P=0.0029$ for difference between doses from baseline to $8 \mathrm{~h}$ ). Average percentages were similar at $24 \mathrm{~h}$, when the neutrophil-platelet aggregates were $13.3 \pm 2.2 \%$ in the rosuvastatin group and $18.1 \pm 3.9 \%$ in the placebo group $(P=0.0109$ for difference between doses from baseline to $24 \mathrm{~h}$ ). Figure $1 \mathrm{~b}$ displays the fold change in neutrophil-platelet aggregates relative to baseline. 


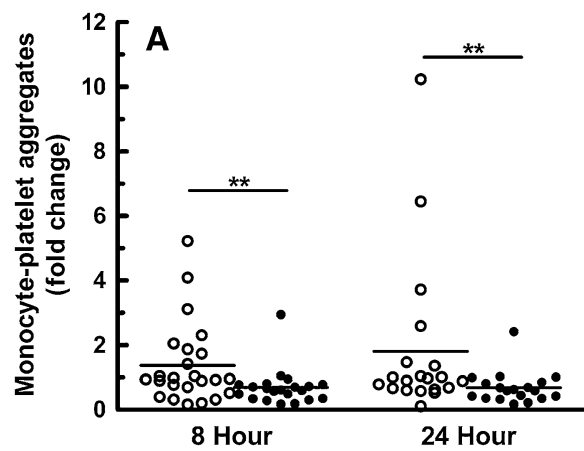

Fig. 1 Platelet-monocyte and platelet-neutrophil aggregates in ACS patients randomized to rosuvastatin or placebo. The fold change from baseline of monocytes with attached platelets for each subject is plotted at 8 and $24 \mathrm{~h}$ following randomization (a). b displays the fold change of neutrophils with attached platelets from baseline. Overall

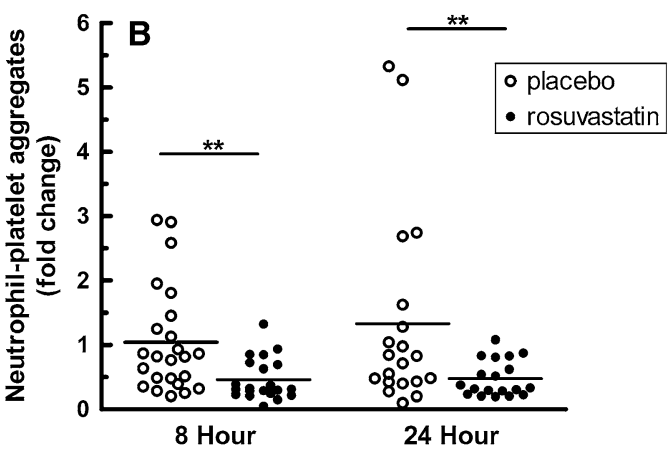

significance between groups over $24 \mathrm{~h}$ is indicated in Table 4 . Significance between the groups at time points are indicated by $(* *)$. Statistical significance was ascertained using a linear mixed model and is indicated in Table 4
Fig. 2 TRAP- or ADP-induced platelet aggregation in ACS patients randomized to rosuvastatin or placebo. Maximum platelet aggregation was measured by light transmission in PRP in response to $15 \mu \mathrm{M}$ TRAP (a) and in response to $5 \mu \mathrm{M}$ ADP (b). Area under the curve values in the Multiplate assay with TRAP (c) or ADP (d) as an agonist. Values are presented as the mean $\pm \mathrm{SD}$ for the groups at the indicated times. Statistical significance of change from baseline for each group was determined using a paired $t$ test
A TRAP
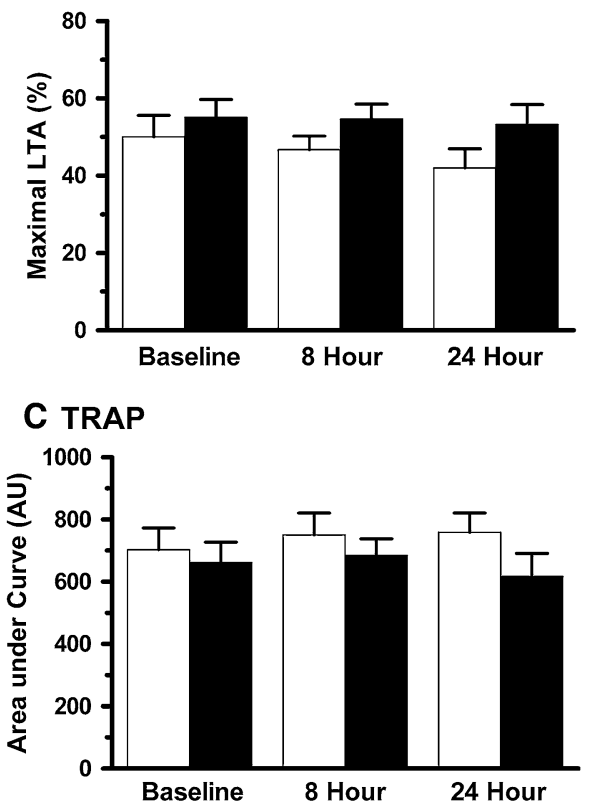

B ADP

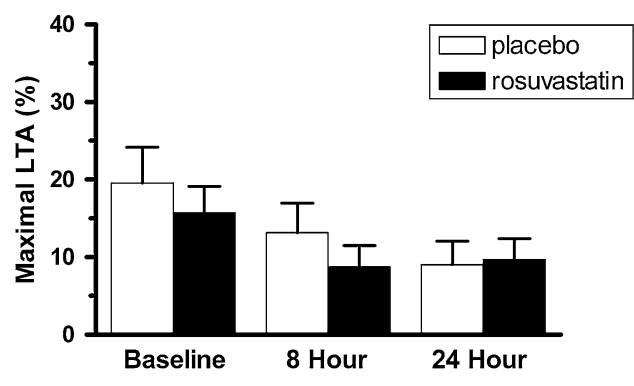

D ADP

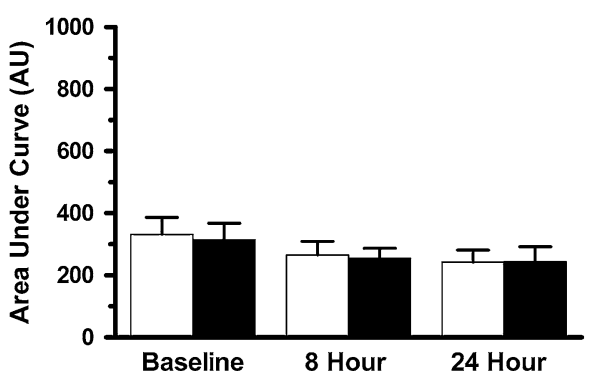

In addition to the primary analysis, an additional analysis was performed by type of event at presentation (NSTEMI/UA and STEMI). In NSTEMI patients, a significant difference in platelet-neutrophil aggregates, but not platelet-monocyte aggregates, occurred at $24 \mathrm{~h}$ in the two groups. STEMI patients had significant difference in the decline over $24 \mathrm{~h}$ in platelet-monocyte aggregates but not platelet-neutrophil aggregates between the groups (Supplemental Fig. 1 and Supplemental Table 2).

In a subset of patients $(n=37)$, the total number of monocyte-platelet and neutrophil-platelet aggregates per $\mu \mathrm{l}$ of blood was determined (Supplemental Table 3). No significant differences were observed between the rosuvastatin and placebo groups at baseline in the numbers of monocyte-platelet or neutrophil-platelet aggregates per $\mu \mathrm{l}$ blood. At $8 \mathrm{~h}$ and $24 \mathrm{~h}$ after rosuvastatin therapy, significant declines occurred in the total number of neutrophil-platelet aggregates/ $\mu \mathrm{l}$ blood $(P=0.0021$ and $P=0.0052$ for 8 and $24 \mathrm{~h}$, respectively). A non-significant trend was observed in monocyte-platelet aggregates $/ \mu \mathrm{l}$ blood $(P=0.0785)$ in the rosuvastatin group at $24 \mathrm{~h}$. No significant changes from baseline were observed in the placebo groups for any aggregate type at 8 or $24 \mathrm{~h}$.

Effect of early high-dose rosuvastatin on platelet function in patients with ACS

Several studies have suggested that statins influence platelet activity in vitro, and the effect of rosuvastatin on leukocyte-platelet aggregates could be the result of inhibition of platelet function. TRAP- and ADP- induced platelet aggregation was therefore monitored prior to and 
up to $24 \mathrm{~h}$ following randomization to rosuvastatin or placebo using both light transmission aggregometry (LTA) and multiple electrode aggregometry (MEA). No significant differences in TRAP-induced LTA were observed between groups, nor were there substantial changes over time (Fig. 2a). At 24 h, ADP-induced LTA was lower than baseline in both groups (Fig. 2b), likely due to treatment with P2Y12 antagonists, but there were no significant differences between groups. Similar results were observed with the MEA assays using TRAP (Fig. 2c) and ADP (Fig. 2d) as agonists, although the response to ADP was less apparent in the MEA assay.

The ability to aggregate is one measure of platelet function. We also examined levels of five biomarkers (sCD40L, P-selectin, VEGF, Platelet Factor 4 (PF4), and RANTES), whose concentrations in plasma maybe affected by platelet activation and secretion. The baseline concentrations of sCD40L and RANTES were significantly higher in both the rosuvastatin and placebo groups in comparison with healthy controls, and the values of both declined over $24 \mathrm{~h}$ with no significant differences between the two groups (Table 5). There were no significant findings for VEGF, P-selectin, and PF4.

Effect of early high-dose rosuvastatin on plasma inflammatory biomarkers in patients with ACS

During vascular inflammation, myeloperoxidase (MPO) is released from leukocytes, particularly neutrophils. Interactions with platelets may promote the release of MPO from neutrophils [13], and the decline in neutrophil-associated MPO in patients with acute MI correlates with an increase in circulating leukocyte-platelet aggregates [13, 14]. Importantly, elevated levels of MPO in patients with chest pain predict ischemic cardiovascular events. We therefore examined MPO in study subjects and results are presented in Fig. 3a and Table 6. At baseline, MPO levels were higher in both groups than in healthy controls. The rosuvastatin group $(P=0.027)$, but not the placebo $(P=0.067)$, group had a significant decrease from baseline to $8 \mathrm{~h}$. By $24 \mathrm{~h}$ following randomization MPO was also significantly lower in the placebo group $(P=0.005)$ and remained significantly lower from baseline in the rousuvastatin group $(P=0.015)$. A similar trend was seen with CRP (Fig. 3b), which increased from baseline to $8 \mathrm{~h}$ in the placebo group $(P=0.050)$ but not in the rosuvastatin group $(P=0.269)$. Both groups had significant increases in plasma CRP $24 \mathrm{~h}$ following randomization.

Table 6 presents information on additional inflammatory cytokines. At baseline, the levels of IL-6, ENA-78, MIP-1 $\beta$, and NAP2 were significantly higher in one or both groups than in healthy controls. IL-6 levels significantly increased at $8 \mathrm{~h}$ in both groups and continued to rise at

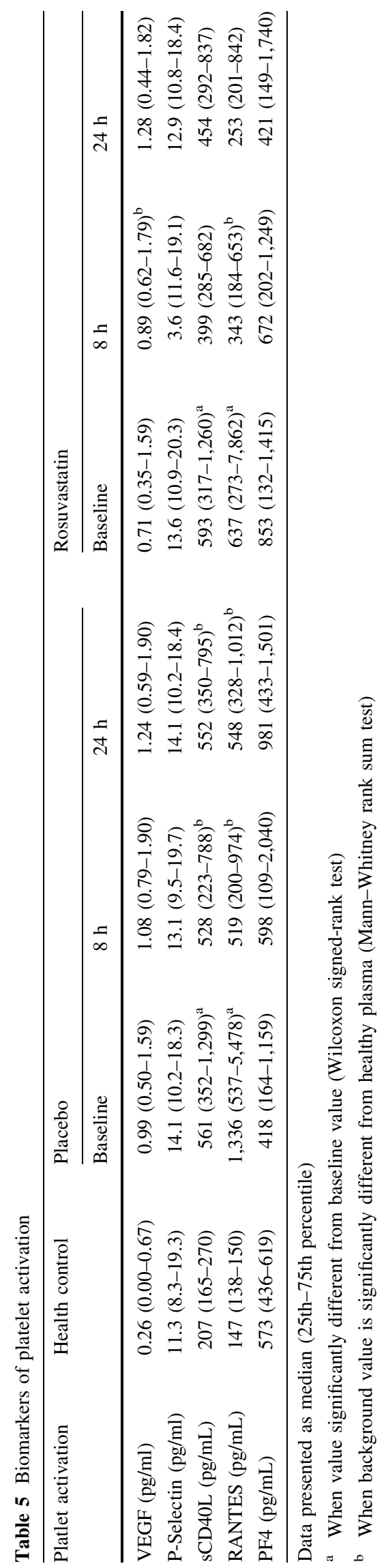



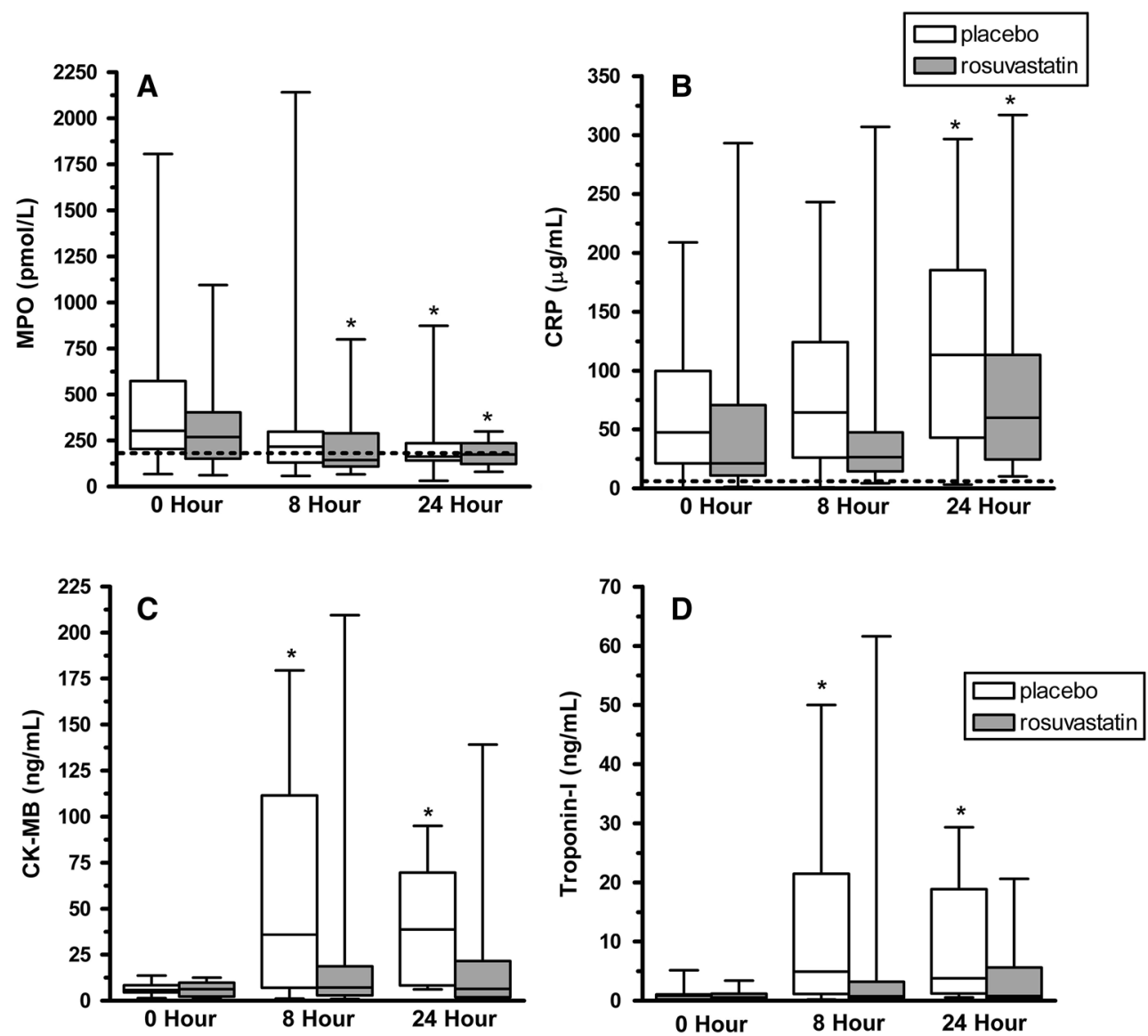

Fig. 3 Inflammatory and cardiac necrosis levels in ACS patients randomized to rosuvastatin or placebo. MPO (a) and CRP (b) levels in subjects randomized to rosuvastatin (shaded boxes) and placebo (white boxes) at the indicated time points. A subpopulation of patients with low baseline values of CK-MB were identified for subsequent cardiac biomarker analysis. CK-MB levels (c) and troponin-I levels (d).

Boxes represent the IQR with the median represented as a solid horizontal line within the box. Whiskers show the extent of the data sets. The dashed lines is the MPO and CRP level in pooled plasma from healthy donors. Statistical significance of change from baseline for each group was determined using a Wilcoxon signed rank test. $P$ values of less than 0.05 are indicated by $(*)$

$24 \mathrm{~h}$ in the rosuvastatin group. ENA-78 and NAP2 decreased at 8 and $24 \mathrm{~h}$ in both groups. MIP- $1 \beta$ remained relatively unchanged in the placebo group at $8 \mathrm{~h}$, with a trend towards reduction at $24 \mathrm{~h}$ in the rosuvastatin group.

Cardiac necrosis markers in patients with ACS

While no significant differences were observed in baseline and peak troponin-I levels between the two groups (Table 3), a post hoc sub-analysis was performed in patients who presented with values $<3 \times$ the upper limit of normal to investigate the effect of rosuvastatin on biomarkers of cardiac necrosis. A total of 24 subjects met this criterion ( $n=12$ in each group). At 8 h, CK-MB levels were elevated significantly from baseline in the placebo group $(P=0.007$, Fig. $3 \mathrm{c})$ but not in the rosuvastatin group $(P=0.176)$. Similarly, there was significant increase in troponin-I levels in the placebo group $(P=0.003$, Fig. $3 \mathrm{~d})$ but not the rosuvastatin group

$(P=0.110)$. At $24 \mathrm{~h}$ troponin-I levels remained significantly higher than baseline in the placebo group $(P=0.037)$ but had not increased significantly from baseline in the placebo group $(P=0.084)$. There were no major adverse ischemic events during the hospital stay for any of the enrolled patients. No major bleeding occurred in any subjects. Within the first 30 days, one subject died due to recurrent MI, and one suffered a stroke. Both individuals had been randomized to the placebo group.

\section{Discussion}

Previous studies have indicated that statins may exert protective effects if taken within $24 \mathrm{~h}$ prior to PCI in patients with ACS [15], although the mechanism is not understood. In the AVATAR study, we demonstrated that intensive statin therapy may be associated with beneficial effects within $8 \mathrm{~h}$ of administration in patients presenting 
with ACS. Early treatment with rosuvastatin $40 \mathrm{mg}$ lowered the percentage of monocytes and neutrophils with attached platelets within $8 \mathrm{~h}$ and was accompanied by a decline in MPO levels. The changes in monocyte-platelet and neutrophil-platelet interactions occurred without a detectable effect of rosuvastatin on platelet aggregation or soluble levels of P-selectin, CD40L, or PF4. Based on subgroup analysis, acute administration of rosuvastatin was associated with reduced biomarkers of cardiac damage within the first $24 \mathrm{~h}$, as has been reported in other settings [16]. Previous studies that observed cardiac necrosis markers following PCI in patients that were pre-loaded with statin demonstrate an acute benefit of statins. Here, we demonstrate acute effects of statins on monocyte-platelet and neutrophil-platelet aggregates in patients presenting with ACS. These findings are also consistent with reports that rosuvastatin reduces platelet-leukocyte aggregate formation in a model of congestive heart failure [17] and blocks postprandial activation of neutrophils [18], although both of these studies examined long term effects of statins, over weeks and did not report immediate effects. The novelty of our study is the identification of an acute effect $(<24 \mathrm{~h})$ of statin therapy on biomarkers of thromboinflammation in the setting of ACS.

Statins work by inhibiting the function of HMG-CoA reductase, which, in turn, lowers the de novo synthesis of cholesterol. Rosuvastatin and other members of the class may have pleiotropic effects that are independent of lowering LDL-cholesterol levels. Our findings are consistent with a rapid mechanism of action independent of LDLcholesterol and suggest that the ability of rosuvastatin, and potentially other statins, to impair platelet-leukocyte interactions could translate into long-term clinical benefit on top of that gained by lowering cholesterol. The AVATAR results are consistent with observations in experimental and in vitro models in which HMG-CoA reductase inhibition attenuated leukocyte-platelet interactions [12, 19]. In vitro, statins reduce the expression of mediators of heterotypic blood cell interactions, such as sCD40, ICAM, and E-selectin [20-22]. Additional mechanisms of effect may include improved endothelial function, decreased oxidative stress, and inhibition thrombogenic responses not measured by platelet aggregation to ADP or thrombin [23].

The precise mechanism by which rosuvastatin reduce platelet-leukocyte interaction remain to be defined. However, much of the data seems to suggest that leukocytes are the cellular targets of the statin. This is in agreement with in vitro studies demonstrating that HMG-CoA reductase inhibitors block Mac-1 activation in monocytes [24]. This is particularly relevant since Mac-1 mediates platelet leukocyte adhesion.

The AVATAR results may also reflect differences in temporal patterns between the rosuvastatin and placebo

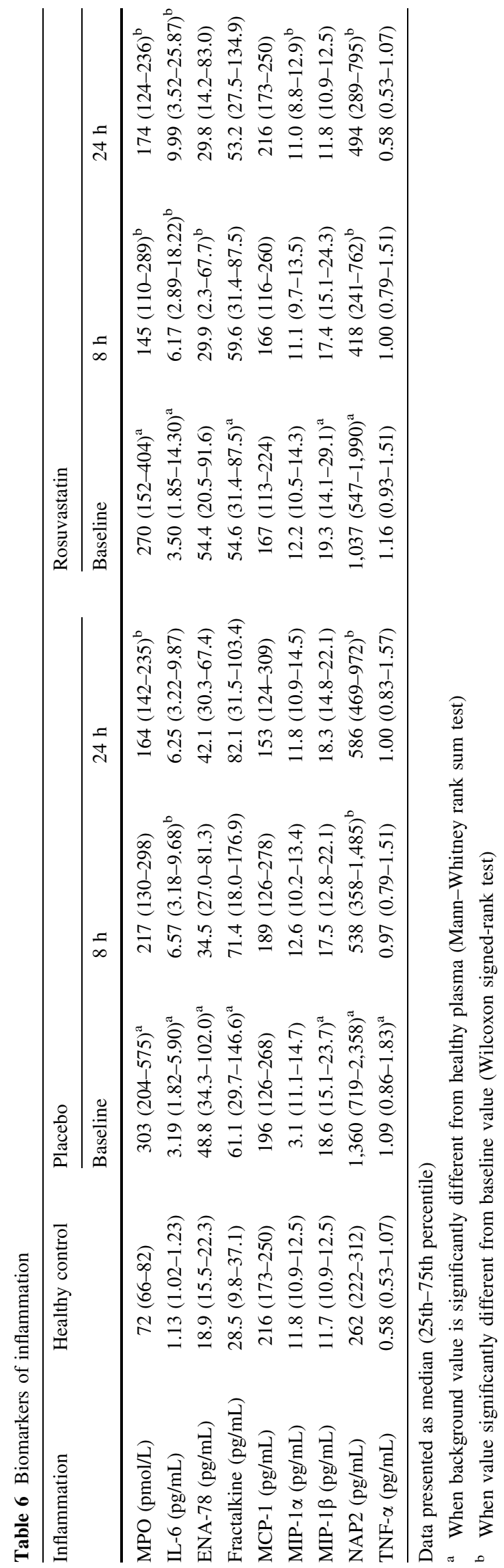


groups. Although no statistically significant difference was observed in overall monocyte-platelet and neutrophilplatelet aggregates at baseline between the two groups, numerically the average percentage of monocytes and neutrophils with attached platelets was higher at baseline in the individuals randomized to rosuvastatin. If these patients were captured at a different time in the presentation of ACS, more rapid reduction in aggregates could have resulted. In that case, we would anticipate that heterotypic aggregates would continue to decline in the placebo group at $24 \mathrm{~h}$, but they did not. Most of the inflammatory biomarkers declined or remain unchanged within the first $24 \mathrm{~h}$ after presentation. The exceptions were IL-6, which was higher at $24 \mathrm{~h}$ in the rosuvastatin group at a time when the frequency of monocyte-platelet and neutrophil-platelet aggregates was reduced, and CRP which increased from baseline at $8 \mathrm{~h}$ in the placebo group but not in the rosuvastatin group.

In summary, the results of the AVATAR trial indicate that targeting pathways that link inflammation and thrombosis may be a beneficial strategy in patients with ACS. Although the sample size was too small to identify an effect on clinical outcomes, when considered with previously published work that demonstrated a reduction in ischemic and clinical events with early high dose statins that associated with reduced biomarkers of cardiac necrosis, our findings suggest that reducing monocyte-platelet and neutrophil-platelet interactions may contribute to the acute benefit that has been observed. If this is true, high-dose statin therapy should be administered rapidly, similarly to aspirin therapy, in patients presenting with ACS to maximize effects independent of LDL-cholesterol lowering.

Acknowledgments This work was supported by an investigatorinitiated Grant from AstraZeneca. TRS was supported in part by T32HL091812 from the Heart Lung and Blood Institute, National Institutes of Health. Additional support was provided by the National Center for Research Resources and the National Center for Advancing Translational Sciences, National Institutes of Health, through Grant UL1TR000117. The content is solely the responsibility of the authors and does not necessarily represent the official views of the NIH.

Disclosure SSS has previously served on advisory boards for AstraZeneca.

Open Access This article is distributed under the terms of the Creative Commons Attribution License which permits any use, distribution, and reproduction in any medium, provided the original author(s) and the source are credited.

\section{References}

1. Levine GN, Bates ER, Blankenship JC, Bailey SR, Bittl JA, Cercek B, Chambers CE, Ellis SG, Guyton RA, Hollenberg SM, Khot UN, Lange RA, Mauri L, Mehran R, Moussa ID, Mukherjee
D, Nallamothu BK, Ting HH (2011) 2011 ACCF/AHA/SCAI Guideline for Percutaneous Coronary Intervention: a report of the American College of Cardiology Foundation/American Heart Association Task Force on Practice Guidelines and the Society for Cardiovascular Angiography and Interventions. Circulation 124:e574-e651

2. Smyth SS, McEver RP, Weyrich AS, Morrell CN, Hoffman MR, Arepally GM, French PA, Dauerman HL, Becker RC (2009) Platelet functions beyond hemostasis. J Thromb Haemost 7:1759-1766

3. Kling D, Stucki C, Kronenberg S, Tuerck D, Rheaume E, Tardif JC, Gaudreault J, Schmitt C (2013) Pharmacological control of platelet-leukocyte interactions by the human anti-P-selectin antibody inclacumab-preclinical and clinical studies. Thromb Res 131:401-410

4. Tardif JC, Tanguay JF, Wright SS, Duchatelle V, Petroni T, Gregoire JC, Ibrahim R, Heinonen TM, Robb S, Bertrand OF, Cournoyer D, Johnson D, Mann J, Guertin MC, L'Allier PL (2013) Effects of the P-selectin antagonist inclacumab on myocardial damage after percutaneous coronary intervention for nonST-segment elevation myocardial infarction: results of the SELECT-ACS trial. J Am Coll Cardiol 61:2048-2055

5. Ridker PM, Fonseca FA, Genest J, Gotto AM, Kastelein JJ, Khurmi NS, Koenig W, Libby P, Lorenzatti AJ, Nordestgaard BG, Shepherd J, Willerson JT, Glynn RJ (2007) Baseline characteristics of participants in the JUPITER trial, a randomized placebo-controlled primary prevention trial of statin therapy among individuals with low low-density lipoprotein cholesterol and elevated high-sensitivity C-reactive protein. Am J Cardiol 100:1659-1664

6. Ridker PM, Danielson E, Fonseca FA, Genest J, Gotto AM Jr, Kastelein JJ, Koenig W, Libby P, Lorenzatti AJ, Macfadyen JG, Nordestgaard BG, Shepherd J, Willerson JT, Glynn RJ (2009) Reduction in C-reactive protein and LDL cholesterol and cardiovascular event rates after initiation of rosuvastatin: a prospective study of the JUPITER trial. Lancet 373:1175-1182

7. Cannon CP, Braunwald E, McCabe CH, Rader DJ, Rouleau JL, Belder R, Joyal SV, Hill KA, Pfeffer MA, Skene AM (2004) Intensive versus moderate lipid lowering with statins after acute coronary syndromes. N Engl J Med 350:1495-1504

8. Schwartz GG, Olsson AG, Ezekowitz MD, Ganz P, Oliver MF, Waters D, Zeiher A, Chaitman BR, Leslie S, Stern T (2001) Effects of atorvastatin on early recurrent ischemic events in acute coronary syndromes: the MIRACL study: a randomized controlled trial. JAMA 285:1711-1718

9. Briguori C, Visconti G, Focaccio A, Golia B, Chieffo A, Castelli A, Mussardo M, Montorfano M, Ricciardelli B, Colombo A (2009) Novel approaches for preventing or limiting events (Naples) II trial: impact of a single high loading dose of atorvastatin on periprocedural myocardial infarction. J Am Coll Cardiol 54:2157-2163

10. Pasceri V, Patti G, Nusca A, Pristipino C, Richichi G, Di Sciascio G (2004) Randomized trial of atorvastatin for reduction of myocardial damage during coronary intervention: results from the ARMYDA (Atorvastatin for Reduction of MYocardial Damage during Angioplasty) study. Circulation 110:674-678

11. Patti G, Pasceri V, Colonna G, Miglionico M, Fischetti D, Sardella G, Montinaro A, Di Sciascio G (2007) Atorvastatin pretreatment improves outcomes in patients with acute coronary syndromes undergoing early percutaneous coronary intervention: results of the ARMYDA-ACS randomized trial. J Am Coll Cardiol 49:1272-1278

12. Yun KH, Jeong MH, Oh SK, Rhee SJ, Park EM, Lee EM, Yoo NJ, Kim NH, Ahn YK, Jeong JW (2009) The beneficial effect of high loading dose of rosuvastatin before percutaneous coronary 
intervention in patients with acute coronary syndrome. Int J Cardiol 137:246-251

13. Caudrillier A, Kessenbrock K, Gilliss BM, Nguyen JX, Marques MB, Monestier M, Toy P, Werb Z, Looney MR (2012) Platelets induce neutrophil extracellular traps in transfusion-related acute lung injury. J Clin Invest 122:2661-2671

14. Maugeri N, Rovere-Querini P, Evangelista V, Godino C, Demetrio M, Baldini M, Figini F, Coppi G, Slavich M, Camera M, Bartorelli A, Marenzi G, Campana L, Baldissera E, Sabbadini MG, Cianflone D, Tremoli E, D'Angelo A, Manfredi AA, Maseri A (2012) An intense and short-lasting burst of neutrophil activation differentiates early acute myocardial infarction from systemic inflammatory syndromes. PLoS ONE 7:e39484

15. Di Sciascio G, Patti G, Pasceri V, Gaspardone A, Colonna G, Montinaro A (2009) Efficacy of atorvastatin reload in patients on chronic statin therapy undergoing percutaneous coronary intervention: results of the ARMYDA-RECAPTURE (Atorvastatin for Reduction of Myocardial Damage During Angioplasty) randomized trial. J Am Coll Cardiol 54:558-565

16. Cay S, Cagirci G, Sen N, Balbay Y, Durmaz T, Aydogdu S (2010) Prevention of peri-procedural myocardial injury using a single high loading dose of rosuvastatin. Cardiovasc Drugs Ther 24:41-47

17. Schafer A, Fraccarollo D, Eigenthaler M, Tas P, Firnschild A, Frantz S, Ertl G, Bauersachs J (2005) Rosuvastatin reduces platelet activation in heart failure: role of NO bioavailability. Arterioscler Thromb Vasc Biol 25:1071-1077

18. van Oostrom AJ, Plokker HW, van Asbeck BS, Rabelink TJ, van Kessel KP, Jansen EH, Stehouwer CD, Cabezas MC (2006) Effects of rosuvastatin on postprandial leukocytes in mildly hyperlipidemic patients with premature coronary sclerosis. Atherosclerosis 185:331-339
19. Kaneider NC, Egger P, Dunzendorfer S, Wiedermann CJ (2002) Rho-GTPase-dependent platelet-neutrophil interaction affected by HMG-CoA reductase inhibition with altered adenosine nucleotide release and function. Arterioscler Thromb Vasc Biol 22:1029-1035

20. Sanguigni V, Pignatelli P, Lenti L, Ferro D, Bellia A, Carnevale R, Tesauro M, Sorge R, Lauro R, Violi F (2005) Short-term treatment with atorvastatin reduces platelet CD40 ligand and thrombin generation in hypercholesterolemic patients. Circulation 111:412-419

21. Alber HF, Frick M, Suessenbacher A, Doerler J, Schirmer M, Stocker EM, Dichtl W, Pachinger O, Weidinger F (2006) Effect of atorvastatin on circulating proinflammatory T-lymphocyte subsets and soluble CD40 ligand in patients with stable coronary artery disease-a randomized, placebo-controlled study. Am Heart J 151:139

22. Patti G, Chello M, Pasceri V, Colonna D, Nusca A, Miglionico M, D’Ambrosio A, Covino E, Di Sciascio G (2006) Protection from procedural myocardial injury by atorvastatin is associated with lower levels of adhesion molecules after percutaneous coronary intervention: results from the ARMYDA-CAMs (Atorvastatin for Reduction of MYocardial Damage during AngioplastyCell Adhesion Molecules) substudy. J Am Coll Cardiol 48:1560-1566

23. Ray KK, Cannon CP (2005) The potential relevance of the multiple lipid-independent (pleiotropic) effects of statins in the management of acute coronary syndromes. J Am Coll Cardiol 46:1425-1433

24. Weber C, Erl W, Weber KS, Weber PC (1997) HMG-CoA reductase inhibitors decrease $\mathrm{CD} 11 \mathrm{~b}$ expression and $\mathrm{CD} 11 \mathrm{~b}-$ dependent adhesion of monocytes to endothelium and reduce increased adhesiveness of monocytes isolated from patients with hypercholesterolemia. J Am Coll Cardiol 30:1212-1217 